\title{
A Directly Numerical Algorithm for a Backward Time-Fractional Diffusion Equation Based on the Finite Element Method
}

\author{
Zhousheng Ruan, ${ }^{1,2,3}$ Zewen Wang, ${ }^{2}$ and Wen Zhang $^{1,2}$ \\ ${ }^{1}$ Fundamental Science on Radioactive Geology and Exploration Technology Laboratory, East China Institute of Technology, \\ Nanchang, Jiangxi 330013, China \\ ${ }^{2}$ School of Science, East China Institute of Technology, Nanchang, Jiangxi 330013, China \\ ${ }^{3}$ School of Mathematics and Statistics, Wuhan University, Wuhan, Hubei 430072, China
}

Correspondence should be addressed to Zewen Wang; zwwang6@gmail.com

Received 8 April 2014; Accepted 22 July 2014

Academic Editor: Valery G. Yakhno

Copyright (C) 2015 Zhousheng Ruan et al. This is an open access article distributed under the Creative Commons Attribution License, which permits unrestricted use, distribution, and reproduction in any medium, provided the original work is properly cited.

We study a backward problem for a time-fractional diffusion equation, which is formulated into a regularized optimization problem. After solving a sequence of well-posed direct problems by the finite element method, a directly numerical algorithm is proposed for solving the regularized optimization problem. In order to obtain a reasonable regularization solution, we utilize the discrepancy principle with decreasing geometric sequence to choose regularization parameters. One- and two-dimensional examples are given to verify the efficiency and stability of the proposed method.

\section{Introduction}

Nowadays, there is increasing attention on fractional diffusion equations which can be used to describe anomalous diffusion phenomena instead of classical diffusion process. These new fractional-order models are more efficient than the integer-order models, because the fractional-order derivatives and integrals enable the description of the memory and hereditary properties of different substance [1]. By an argument similar to the derivation of the classical diffusion equation from Brownian motion, one can derive a fractional diffusion equation from continuous-time random walk. For example, in paper [2] the authors illustrated a fractional diffusion with respect to a non-Markovian diffusion process, while the authors discussed continuous-time random walks on fractals in paper [3].

We notice that mathematical and numerical analysis of the direct problems of the time-fractional diffusion equations has aroused wide concern in recent years; see [410] and references therein. At the same time, the inverse problems for the time-fractional diffusion equations have attracted more and more attention, not only for theoretical analysis but also for popular applications. The authors concluded that there exists a unique weak solution for the backward time-fractional diffusion equation problem under the overdetermined condition $u(x, T) \in H^{2}(\Omega) \cap H_{0}^{1}(\Omega)$ in paper [4]. The authors of papers [11-13] considered the backward problem of the time-fractional diffusion equation and proposed, respectively, a quasi-reversibility method, an optimization method, and a data regularization method for reconstructing the initial value. Inverse source problems for time-fractional diffusion equations were studied by using the method of the eigenfunction expansion [14], the integral equation method [15], and the separation of variables method [16], respectively, for recovering the space-dependent or time-dependent source term. In [17], the authors recovered the temperature function from one measured temperature at one interior point of a one-dimensional semi-infinite fractional diffusion equation based on Dirichlet kernel mollification techniques. The authors studied an inverse problem of identifying a spatially varying potential term in a onedimensional time-fractional diffusion equation from the flux measurements taken at a single fixed time corresponding to a given set of input sources in [18]. Recently, for determining the space-dependent source in a parabolic equation, the authors [19] proposed a regularized optimization method 
together with the linear model function method $[19,20]$ for choosing regularization parameters. Inspired by this noniterative optimization method, we develop it to solve the backward problem for a time-fractional diffusion equation in this paper.

Let $\alpha$ be a constant such that $0<\alpha<1$. We consider the following time-fractional diffusion equation:

$$
\frac{\partial^{\alpha} u(x, t)}{\partial t^{\alpha}}=(L u)(x, t), \quad(x, t) \in \Omega \times(0, T)
$$

with homogeneous boundary condition

$$
\left.u(x, t)\right|_{\partial \Omega}=0, \quad t \in[0, T]
$$

and initial condition

$$
\left.u(x, t)\right|_{t=0}=\varphi(x), \quad x \in \bar{\Omega},
$$

where $\Omega$ is a bounded domain in $R^{d}(d \geq 1)$ and $L$ is symmetric uniformly elliptic operator given by

$$
L(u)=\sum_{i=1}^{d} \frac{\partial}{\partial x_{i}}\left(\sum_{j=1}^{d} \theta_{i, j} \frac{\partial}{\partial x_{j}} u(x)\right)-c(x) u(x) ;
$$

that is, there exists a constant $v>0$, such that $v \sum_{i}^{d} \xi_{i}^{2} \leq$ $\sum_{i, j=1}^{d} \theta_{i, j} \xi_{i} \xi_{j}, x \in \bar{\Omega}$, and $\xi \in \mathbb{R}^{d}$. The coefficients satisfy

$$
\begin{gathered}
\theta_{i, j} \in C^{1}(\bar{\Omega}), \quad \theta_{i, j}=\theta_{j, i}, \\
c(x) \in C(\bar{\Omega}), \quad c(x) \geq 0, \forall x \in \bar{\Omega} .
\end{gathered}
$$

Here, $\partial^{\alpha} u(x, t) / \partial t^{\alpha}$ is the Caputo fractional derivative which is defined by

$$
\begin{aligned}
& \frac{\partial^{\alpha} u(x, t)}{\partial t^{\alpha}} \\
& \quad=\frac{1}{\Gamma(1-\alpha)} \int_{0}^{t}(t-\eta)^{-\alpha} \frac{\partial u}{\partial \eta} \mathrm{d} \eta, \quad 0<\alpha<1,
\end{aligned}
$$

where $\Gamma(1-\alpha)$ is the Gamma function.

If the function $\varphi(x)$ and the coefficients in (1) are all known, problem (1)-(3) is the so-called direct problem that can be solved stably by the finite element method, the finite difference, the spectrum method, and so forth. Here, we focus on the backward problem; that is, we try to determine the initial value $\varphi(x)$ by the additional data $g(x)$ which is the measurement of the exact value $u(x, T)$ and satisfies

$$
\|u(\cdot, T)-g(\cdot)\|_{L^{2}(\Omega)} \leq \delta
$$

for some known error level $\delta>0$. As we all know, the backward problem is ill-posed, which means that the solution does not depend continuously on the given data and any small perturbation in the given data may cause large change to the solution. For overcoming the ill-posedness we will adopt Tikhonov regularization in our treatment.

The rest of the paper is organized as follows. In Section 2, we reformulate the direct problem in a weak and variational sense. Then we formulate the inverse problem into a regularized optimization problem in Section 3. In Section 4, we give implementations of the regularized optimization method. Finally, numerical results are given to illustrate the efficiency and stability of the proposed method.

\section{Weak Form and Weak Solution}

The weak form of problem (1)-(3) is finding $u(\cdot, t) \in H_{0}^{1}(\Omega)$ such that

$$
\begin{aligned}
\left\langle\frac{\partial^{\alpha} u}{\partial t^{\alpha}}, \chi\right\rangle+a(u, \chi) & =0, \quad \forall \chi \in X, 0<t<T, \\
u(0) & =\varphi(x),
\end{aligned}
$$

where $X=H_{0}^{1}(\Omega),\langle\cdot, \cdot\rangle$ denotes the inner product in $L^{2}(\Omega)$, and

$$
a(u, \chi)=\int_{\Omega}\left(\sum_{i, j=1}^{d} \theta_{i, j} \frac{\partial u(x)}{\partial x_{i}} \cdot \chi_{x_{j}}-c(x) u \cdot \chi\right) \mathrm{d} x .
$$

Definition 1. A function $u(x, t)$ is said to be a weak solution of the direct problem (1)-(3) if $u \in C\left([0, T] ; L^{2}(\Omega)\right) \cap C((0, T]$; $\left.H^{2}(\Omega) \cap H_{0}^{1}(\Omega)\right)$ and the weak form $(8)$ is satisfied.

Lemma 2 (see [4]). If $0<\alpha<1, \varphi \in L^{2}(\Omega)$, there exists a unique weak solution $u \in C\left([0, T] ; L^{2}(\Omega)\right) \cap C\left((0, T] ; H^{2}(\Omega) \cap\right.$ $H_{0}^{1}(\Omega)$ ) to problem (1)-(3), and the expression of the weak solution can be formulated by the following eigenfunction expansion:

$$
u(x, t ; \varphi)=\sum_{k=1}^{\infty} E_{\alpha, 1}\left(-\lambda_{k} t^{\alpha}\right)\left(\varphi, \chi_{k}\right) \chi_{k}
$$

where $E_{\alpha, \gamma}(z)$ is the double-parameter Mittag-Leffler function and is defined by

$$
E_{\alpha, \gamma}(z):=\sum_{k=0}^{\infty} \frac{z^{k}}{\Gamma(\alpha k+\gamma)}, \quad z \in \mathbb{C}, \alpha>0, \gamma \geq 0 ;
$$

$\left\{\lambda_{i}\right\}_{j=1}^{\infty}\left(0<\lambda_{1} \leq \lambda_{2} \leq \cdots, i=1, \ldots, \infty\right)$ and $\left\{\chi_{i}\right\}_{j=1}^{\infty}$ are the Dirichlet eigenvalues and the orthonormal eigenfunctions of symmetric uniformly elliptic operator $-L$, respectively.

The following two propositions will be used in the context.

Proposition 3. $E_{\alpha, 1}(-t)$ is a completely monotonic decreasing function for $t>0$ and satisfies

$$
1=\left|E_{\alpha, 1}(0)\right|>\left|E_{\alpha, 1}\left(-\lambda_{1} t^{\alpha}\right)\right|>\left|E_{\alpha, 1}\left(-\lambda_{2} t^{\alpha}\right)\right|>\cdots>0 .
$$

Proposition 4. Let $0<\alpha<2, \beta \in \mathbb{R}$, and $\pi \alpha / 2<\mu<$ $\min \{\pi, \pi \alpha\}$. Then there exists a constant $C=C(\alpha, \beta, \mu)>0$ such that

$$
\left|E_{\alpha, \beta}(z)\right| \leq \frac{C}{1+|z|}, \quad \mu \leq|\arg (z)| \leq \pi, \quad z \in \mathbb{C} .
$$

\section{The Regularized Optimization Problem}

In this section, we will propose a regularized optimization method together with its implementations for solving the considered backward problem. 
3.1. Regularized Optimization Functional. From results of Lemma 2, formula (10) gives a uniquely weak solution $u(x, t ; \varphi)$ for any initial value $\varphi(x) \in L^{2}(\Omega)$. Naturally, it defines a forward operator

$$
M: \varphi(x) \longmapsto u(x, T ; \varphi) .
$$

Clearly, the forward operator $M$ is a linear map and has the following property.

Lemma 5. The operator $M$ is a well-defined bounded linear operator from $L^{2}(\Omega)$ to $L^{2}(\Omega)$. Moreover, it is injective and compact.

Proof. From Lemma 2, the solution $u(x, t ; \varphi)$ can be represented by

$$
u(x, t ; \varphi)=\sum_{k=1}^{\infty} E_{\alpha, 1}\left(-\lambda_{k} t^{\alpha}\right)\left(\varphi, \chi_{k}\right) \chi_{k} .
$$

From Proposition 3, we know that $0 \leq E_{\alpha, 1}(-t)<1$ for $t \in[\sigma,+\infty) ; \sigma$ is a very small positive number. By the orthogonality of $\left\{\chi_{i}\right\}_{j=1}^{\infty}$ and Proposition 4 , we obtain

$$
\begin{aligned}
\|\Delta u(\cdot, t)\|_{L^{2}(\Omega)}^{2} & =\sum_{k=1}^{\infty} \lambda_{k}^{2} E_{\alpha, 1}^{2}\left(-\lambda_{k} t^{\alpha}\right)^{2}\left\langle\varphi, \chi_{k}\right\rangle^{2} \\
& \leq \sum_{k=1}^{\infty} \frac{C^{2}}{\sigma^{2 \alpha}}\left\langle\varphi, \chi_{k}\right\rangle^{2} \\
& =\frac{C^{2}}{\sigma^{2 \alpha}}\|\varphi\|_{L^{2}(\Omega)}^{2}, \quad \forall t \in[\sigma,+\infty),
\end{aligned}
$$

which implies that $\|u\|_{H^{2}(\Omega)} \leq C\|\varphi\|_{L^{2}(\Omega)}$. Then by Sobolev embedding theorem, we conclude the compactness of the operator $M$.

Results of Lemma 5 show that the backward problem is ill-posed due to the compactness of operator $M$. Thus, regularization is necessary for recovering the initial value $\varphi(x)$. To this end, we consider a Tikhonov functional as

$$
J(\varphi)=\frac{1}{2}\|u(x, T ; \varphi)-g\|_{L^{2}(\Omega)}^{2}+\frac{\beta}{2}\|\varphi\|_{L^{2}(\Omega)}^{2}
$$

where $\varphi(x) \in L^{2}(\Omega)$ and $\beta$ is a regularization parameter balancing the fidelity term and the smoothness of the solution. Due to the $L^{2}$-regularization term $(\beta / 2)\|\varphi\|_{L^{2}(\Omega)}^{2}$, the cost functional $J(\varphi)$ is strongly convex. Subsequently, the unique existence of the minimizer can be obtained by standard arguments.

Theorem 6. There exists a unique minimizer $\varphi^{\star}$ to $J(\varphi)$ for any given $\beta>0$.

Now, we formulate the backward problem into the following minimization problem:

$$
\min _{\varphi \in L^{2}(\Omega)} J(\varphi)
$$

3.2. Finite Element Method Approximation. Obviously, problem (18) is a function space minimization problem. Here, we use the finite element method to approximate it. Similar to that done in $[19,21]$, we first triangulate the domain $\Omega$ with a regular triangulation $T_{h}$ of simplicial elements; let $\left\{p_{i}\right\}_{i=0}^{N_{1}}$ be the set of the nodes, and define $V_{h}$ to be the continuous piecewise linear finite element space defined over $T_{h}$; that is,

$$
V_{h}=\left\{v: v \in C_{0}(\Omega),\left.v\right|_{\Delta_{h}} \in P_{1}\left(\Delta_{h}\right), \forall \Delta_{h} \in T_{h}\right\} .
$$

Then any $u_{h} \in V_{h}$ can be repeated as $u_{h}=\sum_{i=0}^{N_{1}} u_{i} \psi_{i}$, where $u_{i}$ is the value of $u_{h}(x)$ at point $p_{i}$, and $\psi_{i}$ is the pyramid function; that is,

$$
\psi_{i}\left(p_{j}\right)= \begin{cases}1, & i=j \\ 0, & i \neq j .\end{cases}
$$

Next, we need to consider the discretization of the bounded linear operator $M$. We will adopt the discrete Galerkin method to solve the direct problem (1)-(3). The time interval $[0, T]$ is partitioned into $N_{2}$ equal subintervals by using nodal points $0=t_{0}<t_{1}<\cdots<t_{N_{2}-1}<t_{N_{2}}=T$, with $t_{k}=k \tau, \tau=T / N_{2}$. Then, the time-fractional derivative $\partial^{\alpha} u(x, t) / \partial t^{\alpha}$ at $t_{k}$ is estimated by

$$
\begin{aligned}
\left.\frac{\partial^{\alpha} u(x, t)}{\partial t^{\alpha}}\right|_{t=t_{k}} & \frac{1}{\Gamma(1-\alpha)} \int_{0}^{t_{k}}\left(t_{k}-\eta\right)^{-\alpha} \frac{\partial u(x, \eta)}{\partial \eta} \mathrm{d} \eta \\
= & \frac{1}{\Gamma(1-\alpha)} \sum_{l=1}^{k} \int_{t_{l-1}}^{t_{l}}\left(t_{k}-\eta\right)^{-\alpha} \frac{\partial u(x, \eta)}{\partial \eta} \mathrm{d} \eta \\
\approx & \frac{\tau^{-\alpha}}{\Gamma(2-\alpha)} \sum_{l=1}^{k}\left(u\left(x, t_{l}\right)-u\left(x, t_{l-1}\right)\right) \\
& \times\left((k+1-l)^{1-\alpha}-(k-l)^{1-\alpha}\right) \\
= & \frac{\tau^{-\alpha}}{\Gamma(2-\alpha)} \sum_{l=1}^{k} \omega_{l}\left(u\left(x, t_{k+1-l}\right)-u\left(x, t_{k-l}\right)\right),
\end{aligned}
$$

where $\omega_{l}=l^{1-\alpha}-(l-1)^{1-\alpha}, l=1,2, \ldots, k, k=1,2, \ldots, N_{2}$. Denote by $u_{h}^{k} \in V_{h}$ the approximation of $u\left(\cdot, t_{k}\right)$ and

$$
D u_{h, t}^{\alpha, k}=\frac{\tau^{-\alpha}}{\Gamma(2-\alpha)} \sum_{l=1}^{k} \omega_{l}\left(u_{h}^{k+1-l}-u_{h}^{k-l}\right) .
$$

Now we define the fully discrete finite element method by

$$
\left\langle D u_{h, t}^{\alpha, k}, \psi\right\rangle+a\left(u_{h}^{k}, \psi\right)=0, \quad u^{0}=\varphi(x)
$$

for any $\psi \in \stackrel{\circ}{V}_{h}$, where $a\left(u_{h}^{k}, \psi\right)=\int_{\Omega}\left(\sum_{i, j=1}^{d} \theta_{i, j}\left(\partial u_{h}^{k} / \partial x_{i}\right) \cdot \psi_{x_{j}}-\right.$ $\left.c(x) u_{h}^{k} \cdot \psi\right) \mathrm{d} x$. The space $\dot{\circ}_{h}$, in which all functions vanish on the boundary $\partial \Omega$, is a subspace of $V_{h}$. Clearly, (23) is a linear system about $u_{h}^{k}, k=1,2, \ldots, N_{2}$. Subsequently, there exists a discrete linear operator $M_{h}$ such that

$$
M_{h}: \varphi \longmapsto u^{k}, \quad k=1,2, \ldots, N_{2} .
$$


Theorem 7. Let $u$ and $u_{h}^{k}$ be the weak solution of (1)-(3) and the discrete Galerkin finite element solution of (23), respectively. Then there is a constant $C>0$ such that, for $0<\alpha<1$,

$$
\left\|u\left(\cdot, t_{k}\right)-u_{h}^{k}\right\|_{L^{2}(\Omega)} \leq C\left(\tau^{2-\alpha}+h\right), \quad k=1,2, \ldots, N_{2},
$$

where $C$ is independent of $h, \alpha$, and $\tau$.

The proof of Theorem 7 follows the same lines as the proof of Theorem 2.1 in [22]. So, we omit it.

\subsection{Implementations of the Regularized Optimization Method.} Applying the interpolation of finite element, the initial value function $\varphi(x)$ can be written approximately in the finite element form of

$$
\varphi \approx \varphi_{h}=\sum_{i=0}^{N_{1}} \varphi_{i} \psi_{i}(x),
$$

where $\varphi_{i}:=\varphi\left(x_{i}\right)$. Due to the linearity of the homogeneous governing equation and the homogeneous boundary condition, we easily see that problem (1)-(3) satisfies the principle of superposition. Here, we also use this principle of superposition to formulate the continuous problem (18) into the following discrete problem:

$$
\begin{aligned}
\min _{\varphi_{h} \in V_{h}} J\left(\varphi_{h}\right)= & \frac{1}{2} \int_{\Omega}\left(\sum_{i=0}^{N_{1}} \varphi_{i} u_{h, i}^{N_{2}}-g_{h}\right)^{2} \mathrm{~d} x \\
& +\frac{\beta}{2} \int_{\Omega}\left(\sum_{i=0}^{N_{1}} \varphi_{i} \psi_{i}(x)\right)^{2} \mathrm{~d} x,
\end{aligned}
$$

where $u_{h, i}^{k}, k=1,2, \ldots, N_{2}$, is the finite element solution of $u(x, t)$ and satisfies

$$
\begin{gathered}
u_{h, i}^{0}=\psi_{i}, \\
\left\langle D u_{h, i}^{\alpha, k}, \psi_{j}\right\rangle+a\left(u_{h, i}^{k}, \psi_{j}\right)=0,
\end{gathered}
$$

for any $\psi_{j} \in S_{h}^{\circ}$ and $k=1,2, \ldots, N_{2}$, where

$$
\begin{gathered}
D u_{h, i}^{\alpha, k}=\frac{\tau^{-\alpha}}{\Gamma(2-\alpha)} \sum_{l=1}^{k} \omega_{l}\left(u_{h, i}^{k+1-l}-u_{h, i}^{k-l}\right), \\
g_{h}(x)=\sum_{i=0}^{N_{1}} g_{i} \psi_{i}(x), \\
a\left(u_{h, i}^{k}, \psi_{j}\right)=\int_{\Omega}\left(\sum_{i, j=1}^{d} \theta_{i, j} \frac{\partial u_{h, i}^{k}}{\partial x_{i}} \cdot \psi_{j_{x_{j}}}-c(x) u_{h, i}^{k} \cdot \psi_{j}\right) \mathrm{d} x .
\end{gathered}
$$

Therefore, numerical solving of the backward problem is essential to determine the $\left(N_{1}+1\right)$-dimensional real vector $\Phi=\left[\varphi_{0}, \ldots, \varphi_{N_{1}}\right]^{T}$.

From the necessary condition for minimizing the approximation function $J\left(\varphi_{h}\right)$, that is,

$$
\frac{\partial J\left(\varphi_{h}\right)}{\partial \varphi_{i}}=0, \quad i=0,1, \ldots, N_{1},
$$

we obtain a linear algebraic system

$$
A \Phi=F
$$

$$
\begin{gathered}
A=\left[\int_{\Omega}\left(u_{h, i}^{N_{2}}(x) u_{h, j}^{N_{2}}(x)+\beta \psi_{i} \psi_{j}\right) d x\right]_{\left(N_{1}+1\right) \times\left(N_{1}+1\right)}, \\
\Phi=\left[\varphi_{0}, \varphi_{1}, \ldots, \varphi_{N_{1}}\right]^{T}, \\
F=\left[\int_{\Omega} g_{h}(x) u_{h, i}^{N_{2}}(x) \mathrm{d} x\right]_{\left(N_{1}+1\right) \times 1} .
\end{gathered}
$$

Let $\Phi^{*}=\left[\varphi_{0}^{*}, \varphi_{1}^{*}, \ldots, \varphi_{N_{1}}^{*}\right]^{T}$ be the solution of (31) for a given regularization parameter $\beta$. Then, we obtain the approximation solution of $\varphi$ as follows:

$$
\varphi_{h}=\sum_{i=0}^{N_{1}} \varphi_{i}^{*} \psi_{i}(x) .
$$

\section{Method for Choosing Regularization Parameters}

As we all know, the backward problem for determining the initial value is an ill-posed problem; that is, the round-off errors and the measurement noises may be highly amplified due to the choice of an unreasonable regularization parameter, therefore making the regularization solution completely useless $[19,20]$. Because of the important role of regularization parameters, a good strategy for selecting regularization parameters should be taken in the computational process. For a fixed $0<r<1$ and $\beta_{0}>0$, we consider a geometric sequence of regularization parameters

$$
\beta_{k}=\beta_{0} r^{k}, \quad k \in \mathbb{N} \text {. }
$$

Then, we employ the discrepancy principle to choose a regularization parameter $\beta_{k^{*}}$ after $k^{*}$ steps with

$$
\begin{array}{r}
\int_{\Omega}\left(u_{h}^{k^{*}}(x, T)-g_{h}\right)^{2} \mathrm{~d} x \leq \delta^{2}<\int_{\Omega}\left(u_{h}^{k}(x, T)-g_{h}\right)^{2} \mathrm{~d} x, \\
0 \leq k<k^{*},
\end{array}
$$

where $u_{h}^{k}(x, t)$ is the finite element solution with respect to $\varphi_{h}$ and $\beta_{k}$.

\section{Numerical Examples}

In all one-dimensional examples, $\Omega=[0,1]$, we divide $\Omega$ into 100 equal subintervals which means that there are 100 elements and 101 nodes, $N_{2}=100,(\mathrm{Lu})(x, t)=$ $(\mathrm{d} / \mathrm{d} x)(\theta(x)(\mathrm{d} / \mathrm{d} x) u)-c(x) u$. In all two-dimensional examples, $\Omega=[0,1] \times[0,1]$, we divide $\Omega$ into 1024 equal triangle element, $N_{2}=80,(L u)(x, t)=\nabla(\theta(x, y) \nabla u)-c(x, y) u$. In the computational process, the measurement vector $g_{h}$ is obtained actually at the points of the mesh grid and added by randomly distributed perturbations with relative noise level $\widehat{\delta}$; that is, $g_{h}=u_{h}(x, T) *\left(1+\widehat{\delta} *\left(2 * \operatorname{rand}\left(\operatorname{size}\left(u_{h}(x, T)\right)\right)-1\right)\right)$. 


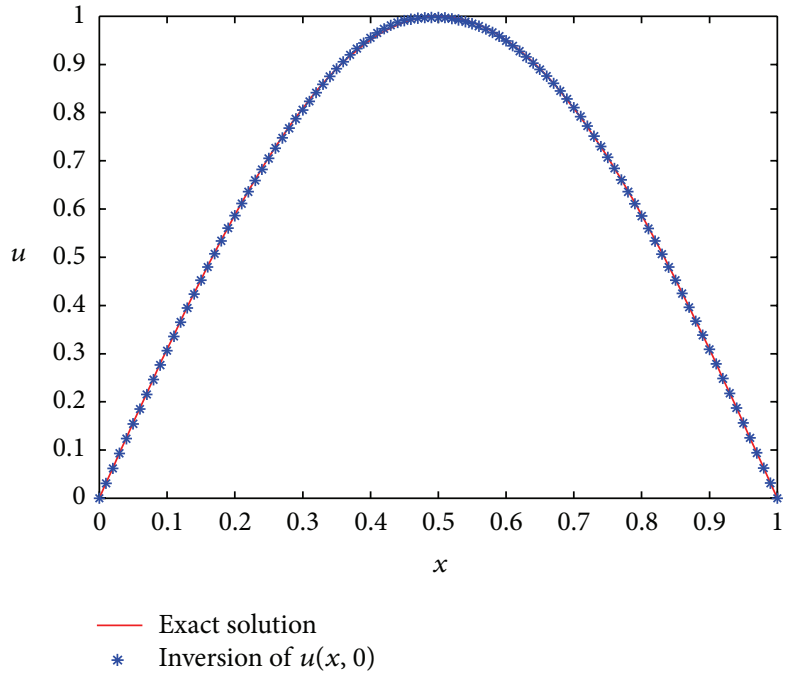

(a) $\widehat{\delta}=0.01$

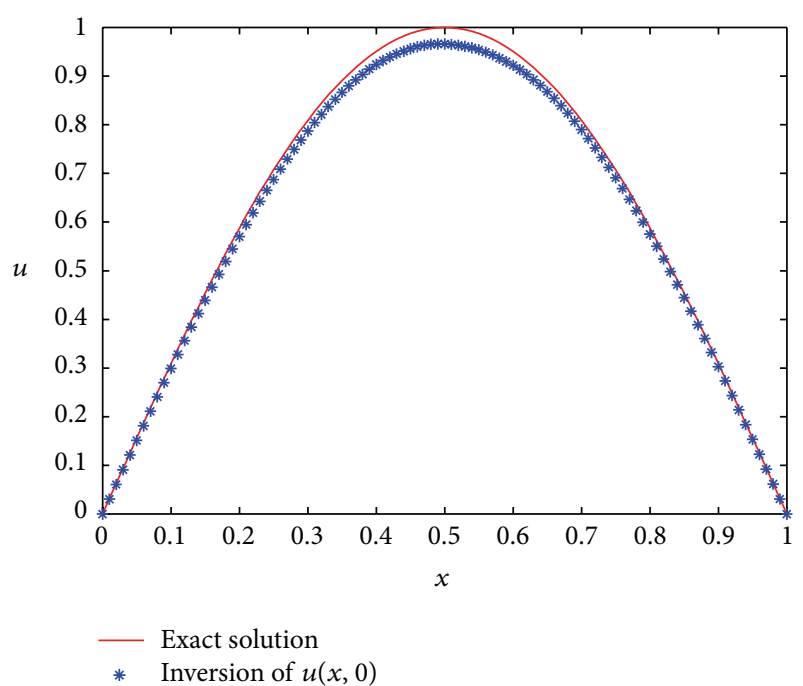

(b) $\widehat{\delta}=0.05$

FIGURE 1: Comparison between exact solution and inverse solution for Example 1.

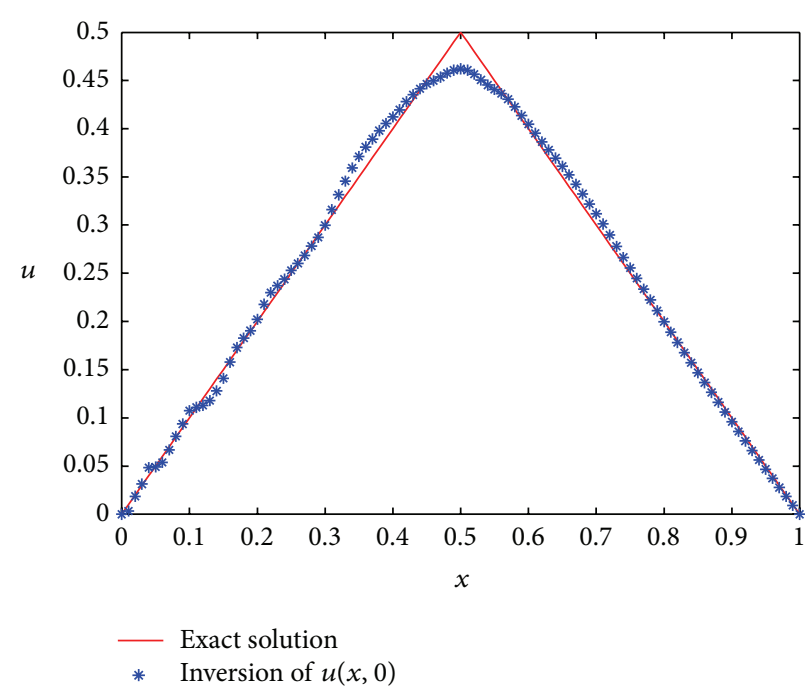

(a) $\widehat{\delta}=0.01$

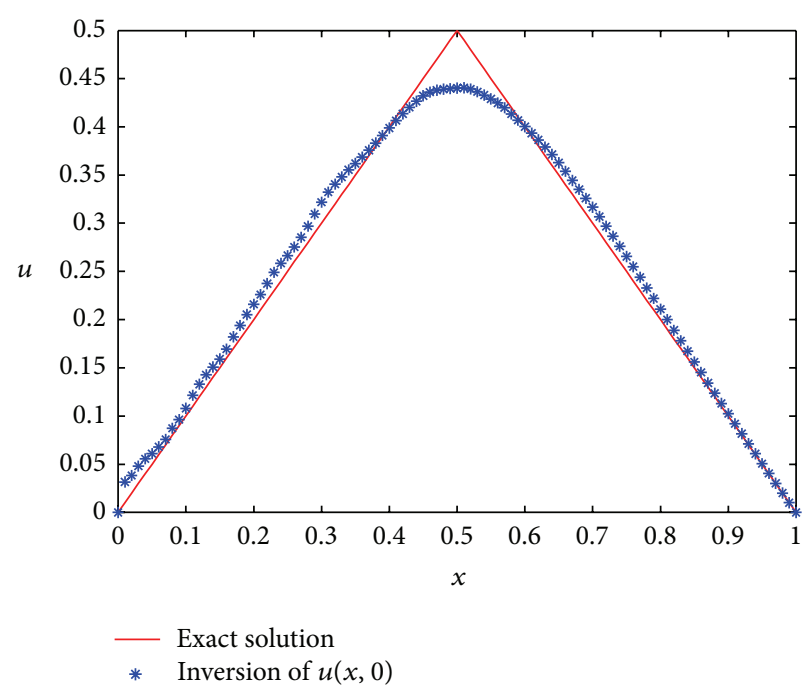

(b) $\widehat{\delta}=0.05$

FIgURE 2: Comparison between exact solution and inverse solution for Example 2.

We take $\beta_{0}=1$ and $r=0.1$ in all numerical examples. The relative error of the inverse solutions is defined by

$$
\text { RelError }=\frac{\left\|\varphi_{\text {exact }}-\varphi_{\text {inversion }}\right\|_{L^{2}(\Omega)}}{\left\|\varphi_{\text {exact }}\right\|_{L^{2}(\Omega)}} .
$$

Example 1. We take $\alpha=0.9, \theta(x)=1$, and $c(x)=0$. Let the exact initial value for problem (1)-(3) be $\sin (\pi x)$. Numerical results for relative noise levels $1 \%$ and $5 \%$ are shown and listed in Figure 1 and Table 1.
Example 2. Let the exact initial value for problem (1)-(3) be

$$
\varphi(x)= \begin{cases}x, & x \in\left[0, \frac{1}{2}\right] \\ (1-x), & x \in\left[\frac{1}{2}, 1\right],\end{cases}
$$

$\theta(x)=x, c(x)=-0.001 x$, and $\alpha=0.6$. Numerical results with the relative noise levels $1 \%$ and 5\% are shown in Figure 2 and listed in Table 1.

Example 3. Let the exact initial value for problem (1)-(3) be $10 x^{3} y^{2}(1-x)(1-y)$. And we take $\alpha=0.8, \theta(x, y)=1$, 


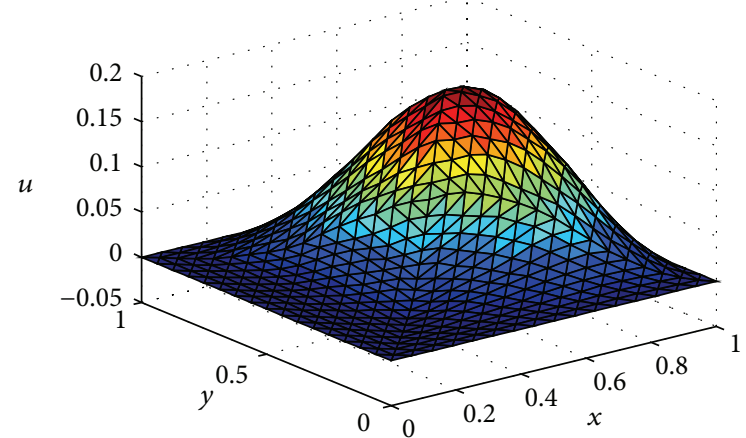

$\square$ Numerical solution

(a) Inverse solution

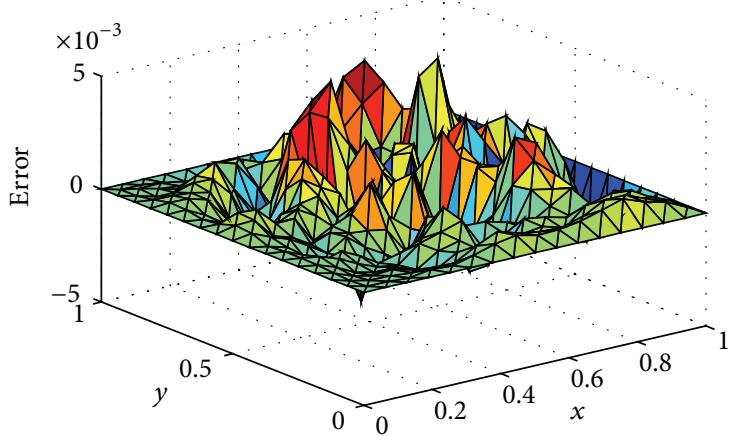

$\square$ Error surface

(b) Error surface

FIgURE 3: Results with $\widehat{\delta}=0.01$ for Example 3 .

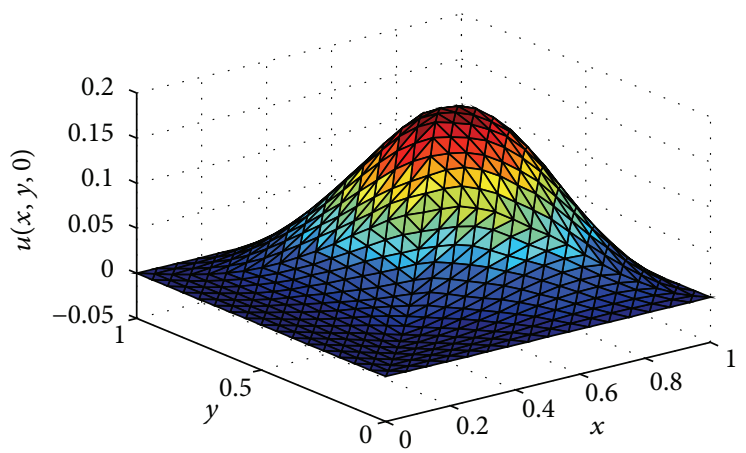

$\square$ Inversion of $u(x, y, 0)$

(a) Inverse solution

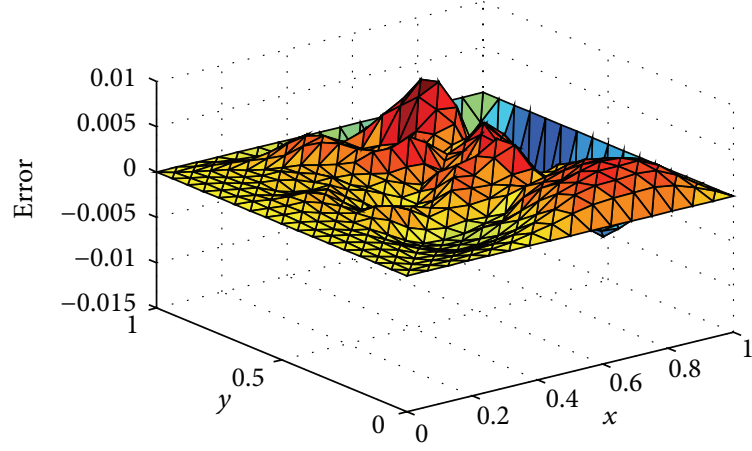

$\square$ Error surface

(b) Error surface

FIgURE 4: Results with $\widehat{\delta}=0.05$ for Example 3 .

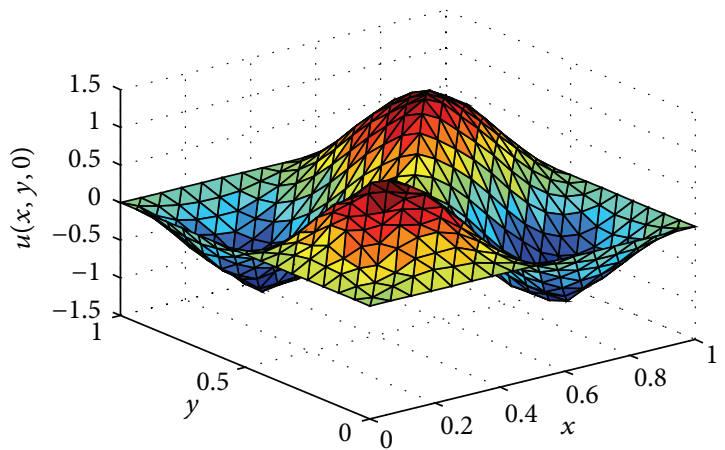

Inversion of $u(x, y, 0)$

(a) Inverse solution

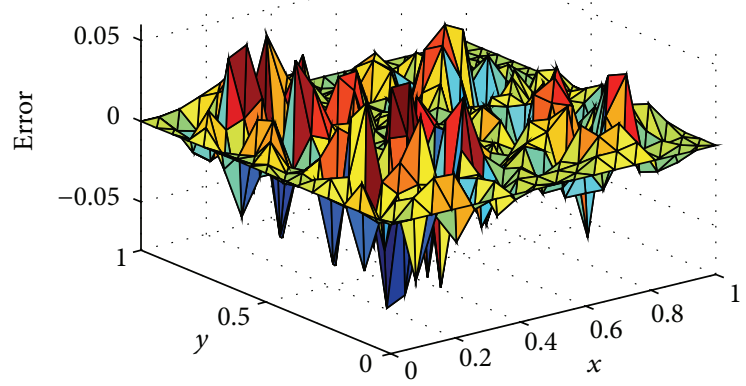

$\square$ Error surface

(b) Error surface

Figure 5: Results with $\widehat{\delta}=0.01$ for Example 4 . 


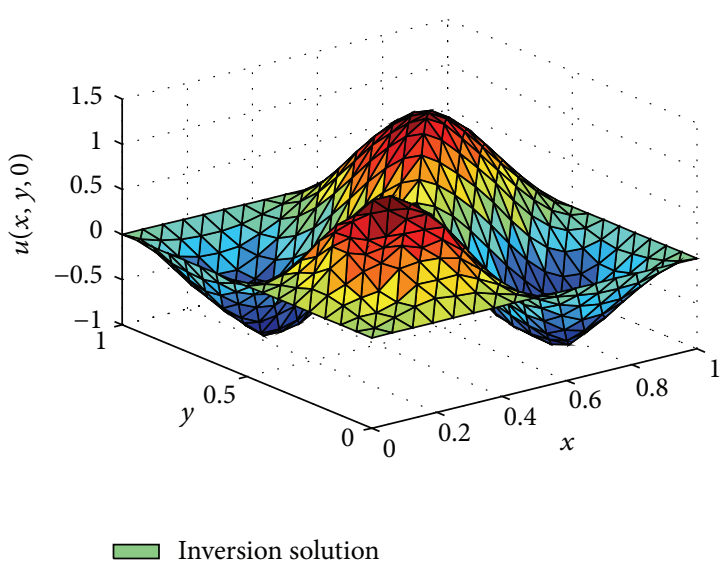

(a) Inverse solution

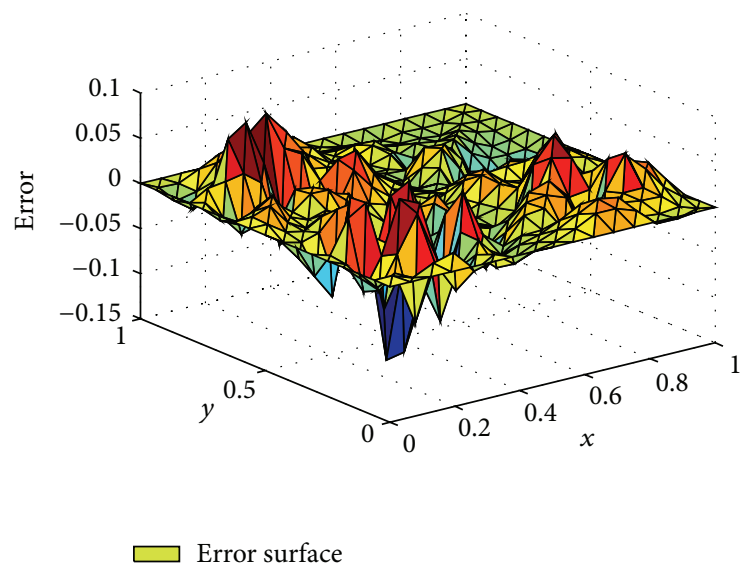

(b) Error surface

FIGURE 6: Results with $\widehat{\delta}=0.05$ for Example 4 .

TABLE 1: Some numerical results for Examples 1-4.

\begin{tabular}{lccc}
\hline Examples & $\widehat{\delta}$ & $\beta$ & RelError \\
\hline \multirow{2}{*}{ Example 1 } & 0.01 & $1 e-6$ & $3.05 e-3$ \\
& 0.05 & $1 e-5$ & $1.61 e-2$ \\
\hline \multirow{2}{*}{ Example 2 } & 0.01 & $1 e-5$ & $2.64 e-2$ \\
& 0.05 & $1 e-4$ & $6.87 e-2$ \\
\hline \multirow{2}{*}{ Example 3 } & 0.01 & $1 e-7$ & $2.29 e-2$ \\
& 0.05 & $1 e-6$ & $6.02 e-2$ \\
\hline \multirow{2}{*}{ Example 4 } & 0.01 & $1 e-3$ & $3.7 e-2$ \\
& 0.05 & $1 e-2$ & $5.01 e-2$ \\
\hline
\end{tabular}

and $c(x, y)=0$. Numerical results are listed in Table 1 and shown in Figures 3 and 4 with relative noise levels 1\% and $5 \%$, respectively.

Example 4. In this example, the exact initial value for problem (1)-(3) is taken as $\varphi(x, y)=\sin (2 \pi x) \sin (2 \pi y)$. And let $\alpha=0.8, \theta(x, y)=0.01(x+y)$, and $c(x, y)=-0.001$. Numerical results are listed in Table 1 and shown in Figures 5 and 6 with relative noise levels $1 \%$ and $5 \%$, respectively.

\section{Conflict of Interests}

The authors declare that there is no conflict of interests regarding the publication of this paper.

\section{Acknowledgments}

This work is supported by National Natural Science Foundation of China (11161002), Young Scientists Training Project of Jiangxi Province (no. 20122BCB23024), Natural Science Foundation of Jiangxi Province of China (no. 20142BAB201008), Ground Project of Science and Technology of Jiangxi Universities (no. KJLD14051), and National High-Tech R\&D Program of China (2012AA061504).

\section{References}

[1] I. Podlubny, Fractional Differential Equations: An Introduction to Fractional Derivatives, Fractional Differential Equations, to Methods of Their Applications, vol. 198 of Mathematics in Science and Engineering, Academic Press, San Diego, Calif, USA, 1999.

[2] R. Metzler and J. Klafter, "Boundary value problems for fractional diffusion equations," Physica A: Statistical Mechanics and its Applications, vol. 278, no. 1-2, pp. 107-125, 2000.

[3] H. E. Roman and P. A. Alemany, "Continuous-time random walks and the fractional diffusion equation," Journal of Physics A Mathematical and General, vol. 27, no. 10, pp. 3407-3410, 1994.

[4] K. Sakamoto and M. Yamamoto, "Initial value/boundary value problems for fractional diffusion-wave equations and applications to some inverse problems," Journal of Mathematical Analysis and Applications, vol. 382, no. 1, pp. 426-447, 2011.

[5] B. Jin, R. Lazarov, and Z. Zhou, "Error estimates for a semidiscrete finite element method for fractional order parabolic equations," SIAM Journal on Numerical Analysis, vol. 51, no. 1, pp. 445-466, 2013.

[6] S. D. Eidelman and A. N. Kochubei, "Cauchy problem for fractional diffusion equations," Journal of Differential Equations, vol. 199, no. 2, pp. 211-255, 2004.

[7] Y. Lin and C. Xu, "Finite difference/spectral approximations for the time-fractional diffusion equation," Journal of Computational Physics, vol. 225, no. 2, pp. 1533-1552, 2007.

[8] F. Mainardi, "The fundamental solutions for the fractional diffusion-wave equation," Applied Mathematics Letters, vol. 9, no. 6, pp. 23-28, 1996.

[9] X. J. Li and C. J. Xu, "A space-time spectral method for the time fractional diffusion equation," SIAM Journal on Numerical Analysis, vol. 47, no. 3, pp. 2108-2131, 2009.

[10] O. P. Agrawal, "Solution for a fractional diffusion-wave equation defined in a bounded domain," Nonlinear Dynamics, vol. 29, no. 1-4, pp. 145-155, 2002.

[11] J. J. Liu and M. Yamamoto, "A backward problem for the timefractional diffusion equation," Applicable Analysis, vol. 89, no. 11, pp. 1769-1788, 2010.

[12] X. T. Xiong, J. X. Wang, and M. Li, "An optimal method for fractional heat conduction problem backward in time," Applicable Analysis, vol. 91, no. 4, pp. 823-840, 2012. 
[13] L. Y. Wang and J. J. Liu, "Data regularization for a backward time-fractional diffusion problem," Computers \& Mathematics with Applications, vol. 64, no. 11, pp. 3613-3626, 2012.

[14] Y. Zhang and X. Xu, "Inverse source problem for a fractional diffusion equation," Inverse Problems, vol. 27, Article ID 035010, pp. 1-12, 2011.

[15] T. Wei and Z. Q. Zhang, "Reconstruction of a time-dependent source term in a time-fractional diffusion equation," Engineering Analysis with Boundary Elements, vol. 37, no. 1, pp. 23-31, 2013.

[16] J. G. Wang, Y. B. Zhou, and T. Wei, “Two regularization methods to identify a space-dependent source for the time-fractional diffusion equation," Applied Numerical Mathematics, vol. 68, pp. 39-57, 2013.

[17] Z.-L. Deng, X.-M. Yang, and X.L. Feng, "A mollification regularization method for a fractional-diffusion inverse heat conduction problem," Mathematical Problems in Engineering, vol. 2013, Article ID 109340, 9 pages, 2013.

[18] B. T. Jin and W. Rundell, "An inverse problem for a onedimensional time-fractional diffusion problem," Inverse Problems, vol. 28, no. 7, Article ID 075010, pp. 1-19, 2012.

[19] Z. Wang and D. Xu, "On the linear model function method for choosing Tikhonov regularization parameters in linear illposed problems," Chinese Journal of Engineering Mathematics, vol. 30, no. 3, pp. 451-466, 2013.

[20] Z. W. Wang and J. J. Liu, "New model function methods for determining regularization parameters in linear inverse problems," Applied Numerical Mathematics, vol. 59, no. 10, pp. 2489-2506, 2009.

[21] Q. Chen and J. J. Liu, "Solving an inverse parabolic problem by optimization from final measurement data," Journal of Computational and Applied Mathematics, vol. 193, no. 1, pp.183203, 2006.

[22] Y. J. Jiang and J. T. Ma, "High-order finite element methods for time-fractional partial differential equations," Journal of Computational and Applied Mathematics, vol. 235, no. 11, pp. 3285-3290, 2011. 


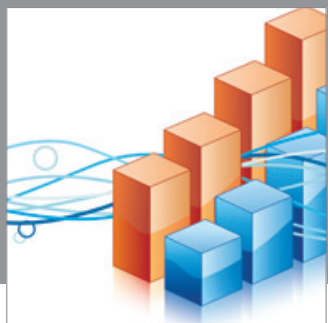

Advances in

Operations Research

mansans

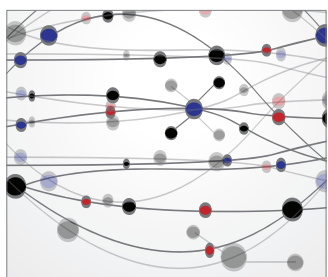

The Scientific World Journal
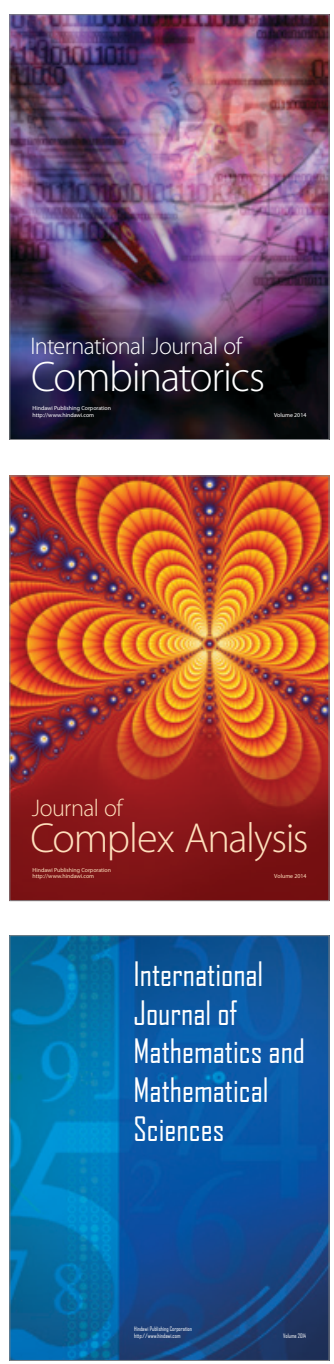
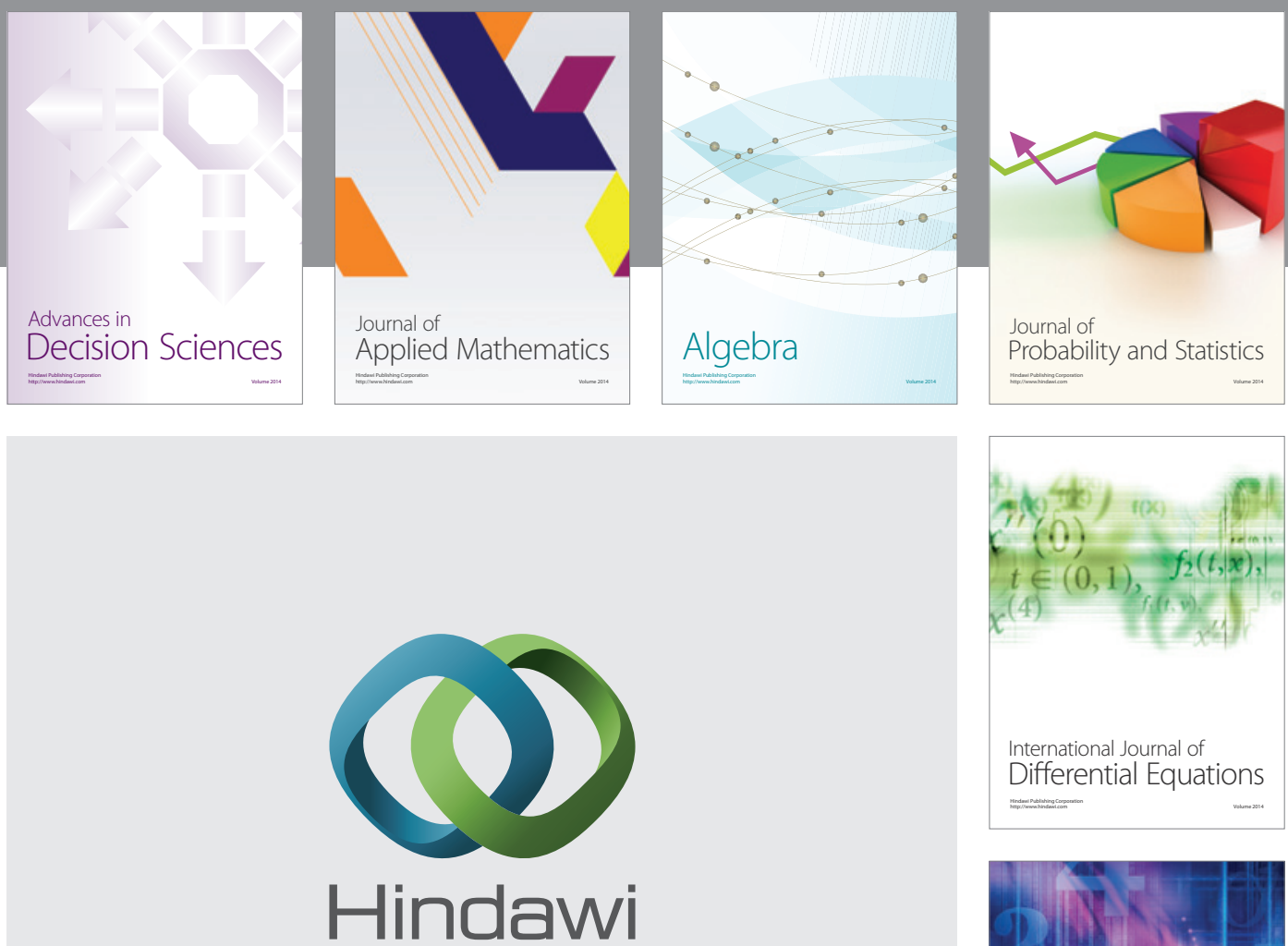

Submit your manuscripts at http://www.hindawi.com
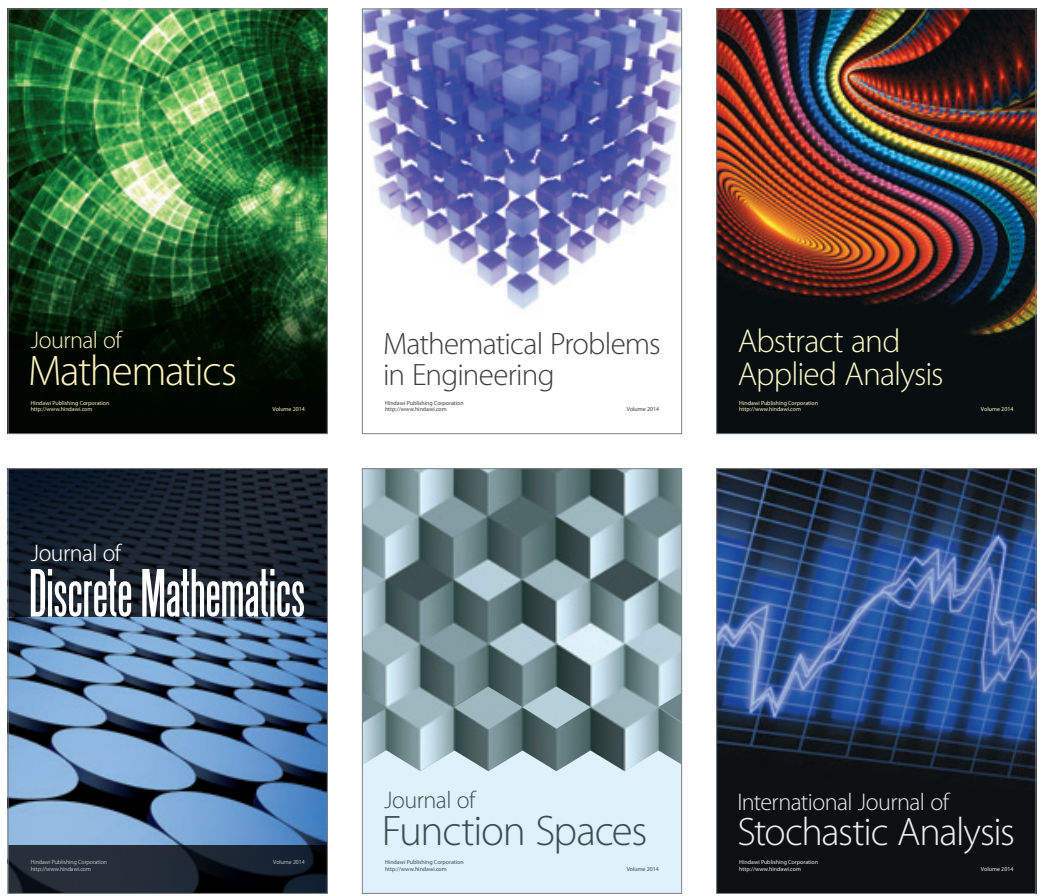

Journal of

Function Spaces

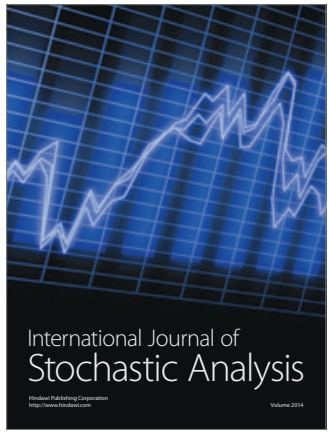

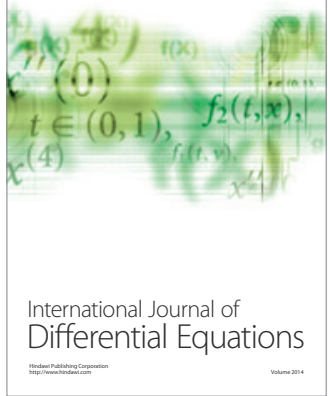
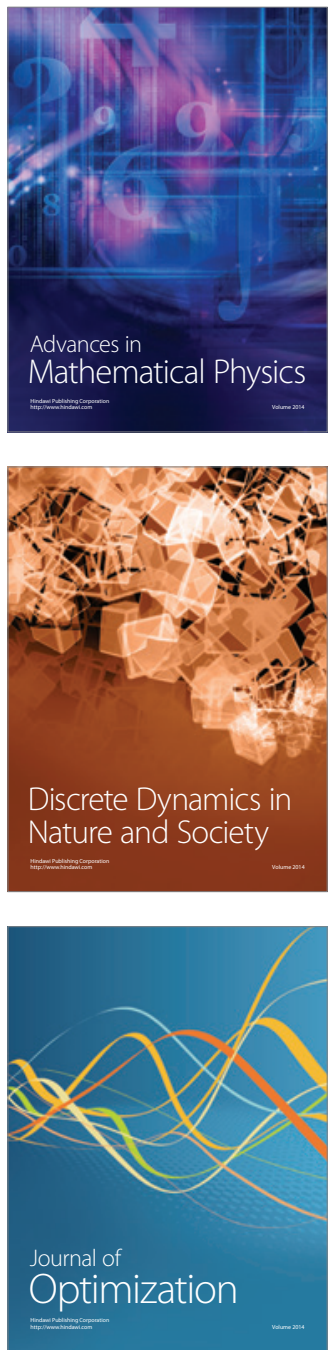